\title{
Long Term Evolution of Magnetic Turbulence in Relativistic Collisionless Shocks
}

\author{
Philip Chang, Anatoly Spitkovsky, and Jonathan Arons \\ Department of Astronomy, Campbell Hall, University of California, Berkeley, CA 94720 \\ Department of Astrophysical Sciences, Peyton Hall, Princeton University, Princeton, NJ 08544 \\ Email: pchang@astro.berkeley.edu, anatoly@astro.princeton.edu,arons@astro.berkeley.edu
}

\begin{abstract}
We study the long term evolution of magnetic fields generated by an initially unmagnetized collisionless relativistic $e^{+} e^{-}$shock. Our 2D particle-in-cell numerical simulations show that downstream of such a Weibel-mediated shock, particle distributions are approximately isotropic, relativistic Maxwellians, and the magnetic turbulence is highly intermittent spatially, nonpropagating, and decaying. Using linear kinetic theory, we find a simple analytic form for these damping rates. Our theory predicts that overall magnetic energy decays like $\left(\omega_{p} t\right)^{-q}$ with $q \sim 1$, which compares favorably with simulations, but predicts overly rapid damping of short wavelength modes. Magnetic trapping of particles within the magnetic structures may be the origin of this discrepancy. We conclude that initially unmagnetized relativistic shocks in electron-positron plasmas are unable to form persistent downstream magnetic fields. These results put interesting constraints on synchrotron models for the prompt and afterglow emission from GRBs.
\end{abstract}

Keywords: shock waves - turbulence - gamma ray: bursts - plasmas

\section{Introduction}

The prompt emission and afterglows of gamma-ray bursts (GRBs) may be manifestations of ultrarelativistic shock waves. These shock waves may be mediated via the relativistic form of Weibel instability (Weibel 1959; Yoon and Davidson 1987; Medvedev and Loeb 1999; Gruzinov and Waxman 1999). The free energy from strong plasma anisotropy in the shock transition layer generates strong magnetic fields (with strengths comparable to the available free energy). However, these fields have very small spatial scales, i.e., the order of the plasma skin depth, $c / \omega_{p}$, where $\omega_{p}$ is the plasma frequency. These initially small-scale B-fields must survive for tens of thousands to millions of inverse plasma periods to serve as this source of the magnetization for synchrotron models of burst emission and afterglows (Gruzinov \& Waxman 1999; Piran 2005ab; Katz, Keshet, \& Waxman 2007). Whether or not these field can is an open question.

Numerical and analytic studies (Kazimura et al. 1998; Silva et al. (2003); Frederiksen et al. 2004; Medvedev et al. 2005; Hededal et al. 2005; Nishikawa et al. 2003, 2005; Spitkovsky this proceedings) have elucidated the basic physics. The instability initially forms filaments of electric current and $B$ fields, which then merge to inverse cascade magnetic energy to larger scales, but only in the foreshock region. When the B-fields reach the magnetic trapping limit (Davidson et al. 1972; Kato 2005; also see Milosavljevic, Nakar, \& Spitkovsky 2006; Milosavlje- vic \& Nakar 2006a), particle orbits become chaotic, disorganizing the filaments. The disorganized magnetic fluctuations scatter their supporting particles, which isotropizes and thermalizes the flow, within tens to hundreds of skin depths (Spitkovsky 2005). The magnetic energy peaks in this layer at $\sim 10-20 \%$ of the bulk plasma flow energy.

However, present simulations have not deeply followed the flow into the downstream region to explore the long term behavior of these B-fields. Thus, the question of the structure and long-term survival of the B-fields remains open (see for instance, Gruzinov \& Waxman 1999; Gruzinov 2001ab; Medvedev et al. 2005).

In this proceeding, we discuss recent work (Chang, Spitkovsky, and Arons 2008; hereafter CSA08) which shows that this magnetic energy must rapidly decay in the downstream medium. We first describe the basic features of the downstream plasma from our numerical simulations. We then calculate the evolution (decay) of the downstream plasma using Vlasov linear response theory and then compare this evolution with simulations. While linear theory does reasonably well in estimating the decay rate of the total magnetic energy, it overestimates the damping rate of shorter wavelength modes. We discuss this discrepancy as a result of magnetic trapping. Finally, we summarize our results. 


\section{Simulation Results}

Spitkovsky (2005, this proceedings) and Spitkovsky and Arons (in prep) describe a series of $2 \mathrm{D}$ and 3D simulations of relativistic shock waves in $e^{+} e^{-}$plasmas. These are Particle-in-Cell (PIC) simulations, using the code TRISTAN-MP. We simulate shocks by injecting cold relativistic plasma particles at one end of a large domain that reflect off a fixed conducting wall at the other end. We use $2 \mathrm{D}$ boxes as large as $50,000 \times 2048$ cells with up to $1.35 \times 10^{10}$ particles to study these shocks. We refer the reader to CSA08 for additional details.

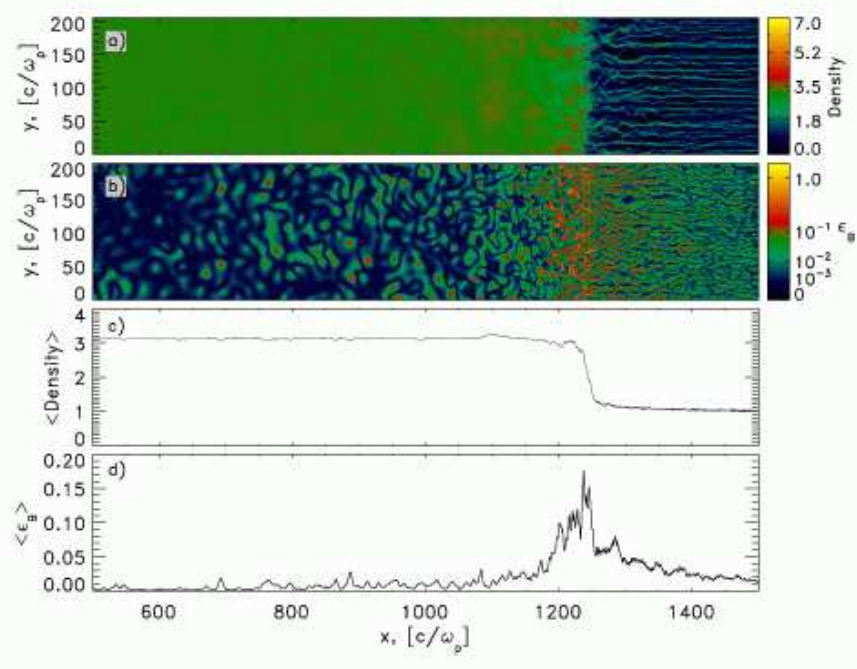

Fig. 1. Snapshot of a region from a large 2D relativistic shock simulation in the downstream frame. a) Density structure, normalized in upstream units, in the simulation plane. b) Magnetic energy, normalized in terms of upstream energy of the incoming flow: $\epsilon_{B}=B^{2} / 4 \pi \gamma_{1} n_{1} m c^{2}$. A power law scaling, $\epsilon_{B}^{1 / 4}$, was applied to stretch the color table to show weak field regions and is reflected in the colorbar. c) Plasma density averaged in the transverse direction as a function of the distance along the flow. d) Magnetic energy density averaged in the transverse direction, as a function of distance along the flow.

Figure 1 shows the snapshots of density and magnetic energy from a typical $2 \mathrm{D}$ simulation. Coordinates are in units of the upstream skin depth, $c / \omega_{p 1}$. In the simulation shown, the upstream flow moves to the left with $\gamma_{1}=15$.

Our simulations are large enough to permit the complete development of the shock and show the main features of contemporary collisionless shock simulations. For instance, we see the factor of $\approx 3.13$ increase in density between the upstream and the downstream (Fig. 1c), which is the expected compression factor (Gallant et al. 1992; Spitkovsky and Arons, in prep). Current filaments show up as an enhancement in the plasma density and magnetic energy density in the foreshock (Fig. 1ab). The scale of the filaments grows towards the shock through merging.

At the shock transition layer, the filaments disorganize and become clumps of magnetic energy. These magnetic clumps lose intensity the further downstream they are from the shock (Figure 1b). We also find that the particle distribution function changes to an isotropic (in the downstream rest frame) thermal population, i.e., the difference between the perpendicular and parallel momentum is extremely small, $<1 \%$. As Figures $1 \mathrm{~b}$ and $\mathrm{d}$ suggest, the magnetic fields decay in the downstream region of the shock.

\section{Downstream Evolution of Magnetic Turbulence}

We now attempt to analytically understand this decay of magnetic turbulence. The simulations show that the downstream plasma is isotropic and the downstream particle distribution function is well described by a relativistic Maxwellian. We will also assume the downstream field amplitudes are so small that particle orbits are almost straight lines.

We begin by deriving the linear plasma response is determined by the plasma susceptibility, $\chi$ (Stix 1992). We evaluate the susceptibilty for distribution functions that are isotropic in two and three dimensions. Details can be found in CSA08. We set $\omega_{r}=0$, because of the non-propagating nature of the magnetic clumps, which we infer from the simulations. In the long wavelength limit, i.e., $k \ll \omega_{p} / c$, where $k$ is the wavenumber, we find:

$$
4 \pi \chi \approx\left\{\begin{array}{ll}
i \frac{\omega_{p}^{2}}{|k| c \omega} & 2 \mathrm{D} \\
i \frac{\pi}{4} \frac{\omega_{p}^{2}}{|k| c \omega} & 3 \mathrm{D}
\end{array} .\right.
$$

Note that the $2 \mathrm{D}$ and $3 \mathrm{D}$ results only vary by a numerical factor. Long wavelength modes have the same qualitative behavior in two and three dimensions.

The plasma susceptibility (eq.[1]) can be utilized to calculate the evolution of an initial field of fluctuations. We refer the interested reader to CSA08 for 
details, but the result is

$$
\frac{d\left|\delta B_{k}\right|^{2}}{d t}=-2 \gamma_{k}\left|\delta B_{k}\right|^{2}
$$

where $\gamma_{k}=(k c)^{2} \omega^{-1} \Im(4 \pi \chi)^{-1}$. The asymptotic forms of $\chi$ from equation (1) for $2 \mathrm{D}$ and $3 \mathrm{D}$ gives

$$
\gamma_{k}=\left\{\begin{array}{l}
\frac{|k c|^{3}}{\omega_{p}^{2}} 2 \mathrm{D} \\
\frac{4}{\pi} \frac{|k c|^{3}}{\omega_{p}^{2}} 3 \mathrm{D} .
\end{array} .\right.
$$

Note the strong cubic $k$ dependence on the decay rate. Short wavelength modes rapidly damp, but longer wavelength modes can persist.

We now compare these expectations to the numerical simulations. We take the Fourier transform of $\delta B$ from our 2D numerical simulations from a downstream region behind the shock front at $x=x_{0}$. We evolve these spectra for 450 (red), 900 (green), and $1350 \omega_{p}^{-1}$ (blue) using equation (3). We compare this analytically evolved spectra to Fourier transformed snapshots taken from our numerical simulations at these times in Figure 2, where $x_{0}=840 c / \omega_{p}$. While theory and simulation agree at very low wavenumber $\left(k_{y} c / \omega_{p} \lesssim 0.2\right)$, theory overpredicts the cutoff in power at larger $\mathrm{k}$. The discrepancy may be due to magnetic trapping (see $\S 4$ ).

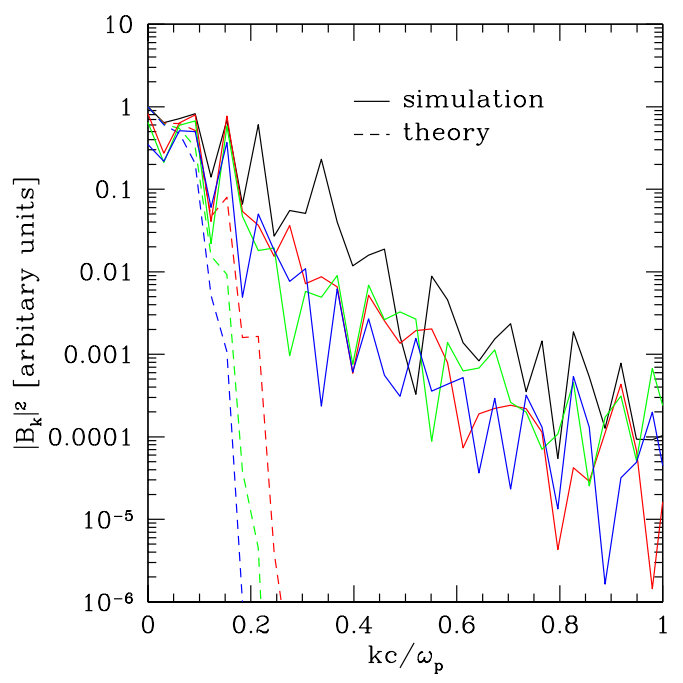

Fig. 2. Spectral evolution of magnetic field from the slice at $840 \mathrm{c} / \omega_{p}$. Initial field spectrum (black solid line) is plotted after $450 \omega_{p}^{-1}$ (red), $900 \omega_{p}^{-1}$ (green), and $1350 \omega_{p}^{-1}$ (blue) based on simulation data. Dashed curves represent analytic evolution of the initial field.

Since total B-field energy is dominated by long wavelength modes, we use equation (2) to find a sim- ple decay law for the total B-field. Again we refer the interested reader to CSA08 for details, but if the initial spatial spectrum is a power law in wave number $\left|\delta B_{k}\right|^{2} \propto k^{2 p}$, then the B-field should decay like

$$
\delta B^{2} \propto t^{-2(p+1) / 3} .
$$

For a shock moving at constant velocity, we have $x_{\text {peak }}-x \propto t$. Hence $\delta B^{2} \propto\left(x_{\text {peak }}-x\right)^{-2(p+1) / 3}$. Our numerical simulations are extremely suggestive that the magnetic energy density follows the a $\delta B^{2} / 8 \pi \propto$ $t^{-2 / 3}$ decay expected for an initially flat magnetic spectrum at early times $(p=0)$, then steepening to a $t^{-1}$ decay at later times $(p=1 / 2)$ as shown in Figure 3. We have analyzed additional simulations with a large transverse spatial scale and they suggest $p=0$. This difference in the index of the decay law expected from the theory and measured from the simulations may also be due to magnetic trapping (see $§ 4$; see Gruzinov 2001b for an alternate explanation and CSA08 for a rebuttal).

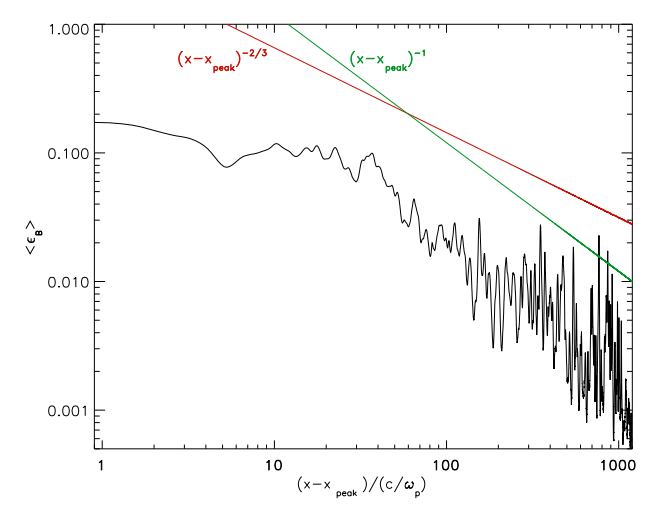

Fig. 3. Magnetic energy density (in units of upstream kinetic energy) as a function of position downstream of the shock. A broken power law proportional $\left(x-x_{\text {peak }}\right)^{-2 / 3}$ fits well at early times, but a $\left(x-x_{\text {peak }}\right)^{-1}$ power law fits better at later times.

\section{Magnetic Trapping}

While our simulations and theory are consistent with one another in suggesting overall power law decay $t^{-q}$ with $q \sim 1$, they disagree for short wavelengths. This discrepancy may arise from the nonlinear effects of magnetic trapping. Our simulations also show that not all particles follow straight line trajectories that are weakly perturbed, but some are partially trapped 
and strongly deflected. By following test particle orbits in our simulations, we find that the Larmor radii of many of the test particles are of the same order of the sizes of these clumps or smaller. These strong departures from weakly perturbed particle dynamics may be the cause of the decreased damping at large wavenumber found in the simulations. It may also modify the overall decay away from $t^{-2 / 3}$ decay law expected from simple linear theory.

\section{Discussion}

We have studied the downstream evolution of magnetic turbulence in the context of a collisionless $e^{+} e^{-}$ shock both analytically and numerically. Our simulations show that the downstream region consist of nonpropagating magnetic clumps embedded in quasihomogenous medium where the background particle distribution function is an isotropic Maxwellian. In such a background, we showed that magnetic energy will decay like $t^{-q}$ with $q \sim 1$. However, linear theory overpredicts the decay rates at short wavelengths compared to simulations. Magnetic trapping may play an role in resolving this discrepancy. Rapid field decay puts severe constraints on GRB emission mechanism, but they may not be inconsistent with GRB observations (see Pe'er \& Zhang 2006). Finally, if ion-electron collisionless shocks reach roughly equipartition with each other as suggest by recent large scale simulations (Spitkovsky $2008)$, they would reproduce the physics of the $e^{ \pm}$ shock and their B-fields would decay as well.

\section{Acknowledgements}

We thank S. Cowley, D. Kocelski, M. Milosavljevic, A. Pe'er and E. Quataert for useful discussions. P.C. is supported by the Miller Institute for Basic Research. J.A. has benefited from the support of NSF grant AST-0507813, NASA grant NNG06GI08G, and DOE grant DE-FC02-06ER41453, all at UC Berkeley; by the Department of Energy contract to the Stanford Linear Accelerator Center no. DE-AC376SF00515; and by the taxpayers of California. A.S. is pleased to acknowledge that the simulations reported on in this paper were substantially performed at the TIGRESS high performance computer center at Princeton University which is jointly supported by the Princeton Institute for Computational Science and Engineering and the Princeton University
Office of Information Technology.

\section{References}

1. Chang, P., Spitkovsky, A., \& Arons, J. 2008, ApJ, 674, 378 (CSA08)

2. Davidson, R. C., Hammer, D. A., Haber, I., \& Wagner, C. E. 1972, Phys. Fluids, 15, 317

3. Frederiksen, J. T., Hededal, C. B., Haugbòlle, T., \& Nordlund, . 2004, ApJ, 608, L13

4. Gallant, Y. A., Hoshino, M., Langdon, A. B., Arons, J., \& Max, C. E. 1992, ApJ, 391, 73

5. Gruzinov, A. 2001a, submitted to ApJ, astro$\mathrm{ph} / 0111321$

6. Gruzinov, A. 2001b, ApJ, 563, L15

7. Gruzinov, A. \& Waxman E. 1999, ApJ, 511, 852

8. Hededal, C. B., Trier Frederiksen, J., Haugboelle, T., \& Nordlund, A. 2005, Neutrinos and Explosive Events in the Universe, Proceedings of the 14th Course of the International School of Cosmic Rays Astrophysics, a NATO Advanced Study Institute, held in Erice, Italy, 2-13 July 2004. Edited by Maurice M. Shapiro, Stanev Todor, and John P. Wefel.

9. Kato, T. N. 2005, Phys. Plasmas, 12, 80705

10. Katz, B., Keshet, U., \& Waxman, E. 2007, ApJ, 655, 375

11. Kazimura, Y., Sakai, J. I., Neubert, T., \& Bulanov, S. V. 1998, ApJ, 498, L183

12. Medvedev, M. V. \& Loeb, A. 1999, ApJ, 526, 697

13. Medvedev, M. V., Fiore, M., Fonseca, R. A., Silva, L. O.; Mori, W. B. 2005, ApJ, 618, L75

14. Milosavljevic, M., Nakar, E., \& Spitkovsky, A. 2006, ApJ, 637, 765

15. Milosavljevic, M. \& Nakar, E. 2006a, ApJ, 641, 978

16. Mikhailovskii, A. B. 1979, Plasma Phys., 22, 133

17. Nishikawa, K.-I., Hardee, P., Richardson, G., Preece, R., Sol, H., \& Fishman, G. J. 2003, ApJ, 595, 555

18. Nishikawa, K.-I., Hardee, P., Richardson, G., Preece, R., Sol, H., \& Fishman, G. J. 2005, ApJ, 622, 927

19. Pe'er, A. \& Zhang, B. 2006, ApJ, 653, 454

20. Piran, T. 2005a, Rev. of Modern Phys., 76, 1143

21. Piran, T. 2005b, Magnetic Fields in the Universe, Angra dos Reis, Brazil, Nov. 29-Dec 3, 2004, Ed. E. de Gouveia del Pino, AIP Conference Proceedings, v784 (New York:AIP), p164

22. Silva, L. O., Fonseca, R. A., Tonge, J. W., Dawson, J. M., Mori, W. B., \& Medvedev, M. V. 2003, ApJ, 596,121

23. Spitkovsky, A. 2005, AIP Conf. Proc, 801, 345; astro$\mathrm{ph} / 0603211$

24. Spitkovsky, A. 2008, ApJ, 673, L38

25. Stix, T.H. 1992, "Waves in Plasmas" (American Institute of Physics: New York)

26. Weibel, E. S. 1959, Phys. Rev. Letts., 2, 83

27. Yoon, P. H., \& Davidson, R. C. 1987, Phys. Rev. A., 35,2718 